\title{
Traditional Chinese Medicine Use among Patients with Psoriasis in Taiwan: A Nationwide Population-Based Study
}

\author{
Shu-Wen Weng, ${ }^{1,2}$ Bor-Chyuan Chen, ${ }^{3}$ Yu-Chiao Wang, ${ }^{4}$ Chun-Kai Liu, ${ }^{5}$ \\ Mao-Feng Sun, ${ }^{1,5,6,7}$ Ching-Mao Chang, ${ }^{8,9}$ Jaung-Geng Lin, ${ }^{1,6}$ and Hung-Rong Yen,5,6,7,10 \\ ${ }^{1}$ Graduate Institute of Chinese Medicine, College of Chinese Medicine, China Medical University, Taichung, Taiwan \\ ${ }^{2}$ Department of Chinese Medicine, Taichung Hospital, Ministry of Health and Welfare, Taichung, Taiwan \\ ${ }^{3}$ Department of Chinese Medicine, Dalin Tzu Chi Hospital, Buddhist Tzu Chi Medical Foundation, Chiayi, Taiwan \\ ${ }^{4}$ Management Office for Health Data, China Medical University Hospital, Taichung, Taiwan \\ ${ }^{5}$ Department of Chinese Medicine, China Medical University Hospital, Taichung, Taiwan \\ ${ }^{6}$ School of Chinese Medicine, College of Chinese Medicine, China Medical University, Taichung, Taiwan \\ ${ }^{7}$ Research Center for Chinese Medicine \& Acupuncture, China Medical University, Taichung, Taiwan \\ ${ }^{8}$ Center for Traditional Medicine, Taipei Veterans General Hospital, Taipei, Taiwan \\ ${ }^{9}$ Graduate Institute of Clinical Medicine and Graduate Institute of Traditional Chinese Medicine, College of Medicine, \\ Chang Gung University, Taoyuan, Taiwan \\ ${ }^{10}$ Research Center for Traditional Chinese Medicine, Department of Medical Research, China Medical University Hospital, \\ Taichung, Taiwan
}

Correspondence should be addressed to Jaung-Geng Lin; jglin@mail.cmu.edu.tw and Hung-Rong Yen; hungrongyen@gmail.com

Received 11 July 2016; Accepted 5 September 2016

Academic Editor: Ki-Wan Oh

Copyright @ 2016 Shu-Wen Weng et al. This is an open access article distributed under the Creative Commons Attribution License, which permits unrestricted use, distribution, and reproduction in any medium, provided the original work is properly cited.

\begin{abstract}
Traditional Chinese medicine (TCM) has long been used for patients with psoriasis. This study aimed to investigate TCM usage in patients with psoriasis. We analyzed a cohort of one million individuals representing the 23 million enrollees randomly selected from the National Health Insurance Research Database in Taiwan. We identified 28,510 patients newly diagnosed with psoriasis between 2000 and 2010. Among them, 20,084 (70.4\%) patients were TCM users. Patients who were female, younger, white-collar workers and lived in urbanized area tended to be TCM users. The median interval between the initial diagnosis of psoriasis to the first TCM consultation was 12 months. More than half $(N=11,609 ; 57.8 \%)$ of the TCM users received only Chinese herbal medicine. Win-qing-yin and Bai-xian-pi were the most commonly prescribed Chinese herbal formula and single herb, respectively. The core prescription pattern comprised Mu-dan-pi, Wen-qing-yin, Zi-cao, Bai-xian-pi, and Di-fu-zi. Patients preferred TCM than Western medicine consultations when they had metabolic syndrome, hepatitis, rheumatoid arthritis, alopecia areata, Crohn's disease, cancer, depression, fatty liver, chronic airway obstruction, sleep disorder, and allergic rhinitis. In conclusion, TCM use is popular among patients with psoriasis in Taiwan. Future clinical trials to investigate its efficacy are warranted.
\end{abstract}

\section{Introduction}

Psoriasis is a common chronic immune-mediated inflammation disease. The prevalence rates of psoriasis ranged from $0.7 \%$ to $2.9 \%$ in Europe, 0.7 to $2.6 \%$ in the United States [1], and $0.235 \%$ in Taiwan [2]. The burden of psoriasis was estimated as $\$ 35.2$ billion in 2013 in the United States [3], while the estimated annual total cost for psoriasis was $\$ 53.8$ million in Taiwan [4]. The cost of long-term therapy and social economic burden of psoriasis have posed a significant impact on healthcare system.

Current treatment for psoriasis is not fully satisfactory. Several types of dermatological treatment are available, ranging from corticosteroids, vitamin D analogs, and phototherapy for mild-moderate psoriasis to retinoids, methotrexate, cyclosporine, apremilast, and biologic immune modifying agents for severe psoriasis [5]. However, many patients are concerned about the side effects of these treatments. 
Skin atrophy may be induced by long-term use of topical corticosteroids [6]. Common adverse events of topical steroid and vitamin $\mathrm{D}$ agent may be partial local irritation and skin pain [7]. Apremilast may cause nausea, upper respiratory tract infection, and diarrhea [8]. Phototherapy may increase the risk of skin cancer [9]. Methotrexate was associated with the increased risk of liver injury [10]. Oral cyclosporin may have the risk of kidney toxicity [11]. A web-based survey study showed that patients with psoriasis were moderately satisfied with their treatment [12].

Traditional Chinese medicine (TCM), which includes acupuncture and moxibustion, Chinese traumatology, and Chinese herbal medicine (CHM), has been integrated as an important part of healthcare in Taiwan. It has been commonly used for dermatitis [13], gastrointestinal disease [14], rheumatoid arthritis [15], diabetic mellitus [16, 17], gynecological disorder [18], and cancer [19, 20] patients. Previous studies showed that CHM plus acitrerin had add-on effect [21] and Chinese herbal bath combined with phototherapy was superior to phototherapy alone [22] for psoriasis. Topical application of Lindioil, extract of Qing-dai (Indigo Naturalis; Baphicacanthus cusia (Nees) Bremek, Polygonum tinctorium Aiton, Isatis indigotica Fortune ex Lindl.) in oil, was effective for treating nail psoriasis [23] and Chinese herbal ointment that contains Qing-dai was effective for plaque-type psoriasis [24].

In the United States, $2.0 \%$ of patients with psoriasis received complementary and alternative medicine (CAM) therapy [25]. Despite the growing interests in utilizing CAM, there remains a critical knowledge gap on the ethnopharmacological analysis of the TCM prescription patterns for psoriasis patients.

In Taiwan, the compulsory National Health Insurance (NHI) system was launched in 1995 and the NHI program started to reimburse TCM service in 1996. As of 2015, The NHI program covered $99.6 \%$ of Taiwanese population [26]. To investigate the prescription patterns of CHM for psoriasis patients, we took advantage of the National Health Insurance Research Database (NHIRD), which contains registration files and claims data for reimbursement. We analyzed a cohort of one million beneficiaries from the NHIRD from 2000 to 2010. This study is important to delineate the TCM utilization patterns among patients with psoriasis. It could be regarded as a consensus of TCM formulas/herbs for further pharmacological investigation or clinical trials.

\section{Material and Methods}

2.1. Data Source. This study used datasets from the NHIRD (http://nhird.nhri.org.tw/en/) in Taiwan as our previous reports $[14,18]$. The NHI program in Taiwan reimbursed TCM services (CHM, acupuncture/moxibustion, and Chinese traumatology therapy) provided by licensed TCM doctors. The NHI database consists of registration files and original claim data for reimbursement. The large-scale computerized data derived from the NHI program were maintained by the National Health Research Institutes, Taiwan, and provided to scientists in Taiwan for research purposes. All registration data in the NHIRD consist of demographic characteristics, diagnosis, clinical visits, hospitalization, procedures, prescriptions, and the medical costs for reimbursement [27]. The diagnostic codes were in the International Classification of Diseases, Ninth Revision, Clinical Modification (ICD-9$\mathrm{CM}$ ) formats.

2.2. Study Population and Variables. The flow chart of enrolling psoriasis patients was shown in Figure 1. A one million random sample from the NHIRD was selected for this study. All the patients $(n=28,510)$ with newly diagnosed psoriasis (ICD-9-CM code: 696) between January 2000 and December 2010 were included in this study and then followed up until the end of 2011. After a confirmed diagnosis with psoriasis, those consulted with TCM doctors were grouped as TCM users $(n=20,084)$ and the others as non-TCM users $(n=8426)$. We investigated the demographic characteristics of TCM users and non-TCM users, including sex, age, occupation, urbanization, and the time between being diagnosed of psoriasis and TCM treatment. We analyzed the incidence rate ratio of diseases between TCM and non-TCM users. We also analyzed ten most common herbal formulas and single herbs prescribed by TCM doctors for psoriasis patients. Herbal formulas were listed in pin-yin name and English name. Single herbs were listed in pin-yin name, Latin name, and botanical plant name. TCM indications of the Chinese herbal formulas and single herbs were based on TCM theory $[28,29]$. Full botanical names comply with the International Plant Names List (IPNI; http://www.ipni.org/) and The Plant List (http://www.theplantlist.org/) [30].

2.3. Core Patterns of Chinese Herbal Medicine. The core pattern of CHM used in treating psoriasis patients was identified with an open-sourced freeware NodeXL (http://nodexl .codeplex.com/), and all the selected two drugs combinations were applied in this network analysis. The line width, ranging from 1 to 5 in the network figure, was defined by counts of connections between a CHM and coprescribed CHM, and thicker widths of line connections indicated a significant prescription pattern [31]. The network analysis manifested the core pattern of the top 50 two-drug combinations in this survey.

2.4. Ethical Considerations. All of the information that could be used to identify individuals or care providers were deidentified and encrypted before release. It is not possible to identify any individuals or care providers at any level in this database. This study was approved by the Research Ethics Committee of China Medical University and Hospital (CMUH104-REC2-115).

2.5. Statistical Analysis. We used the SAS software, version 9.4 (SAS Institute Inc., Cary, NC, USA), to analyze the datasets retrieved from the NHIRD. Descriptive statistics was applied to determine the demographic characteristics, treatment modalities, and the frequency of prescribed herbal formulas and single herbs. The diagnoses were coded according to the International Classification of Diseases, Ninth Revision, Clinical Modification (ICD-9-CM) codes. To present the overall structure of the study groups, we 


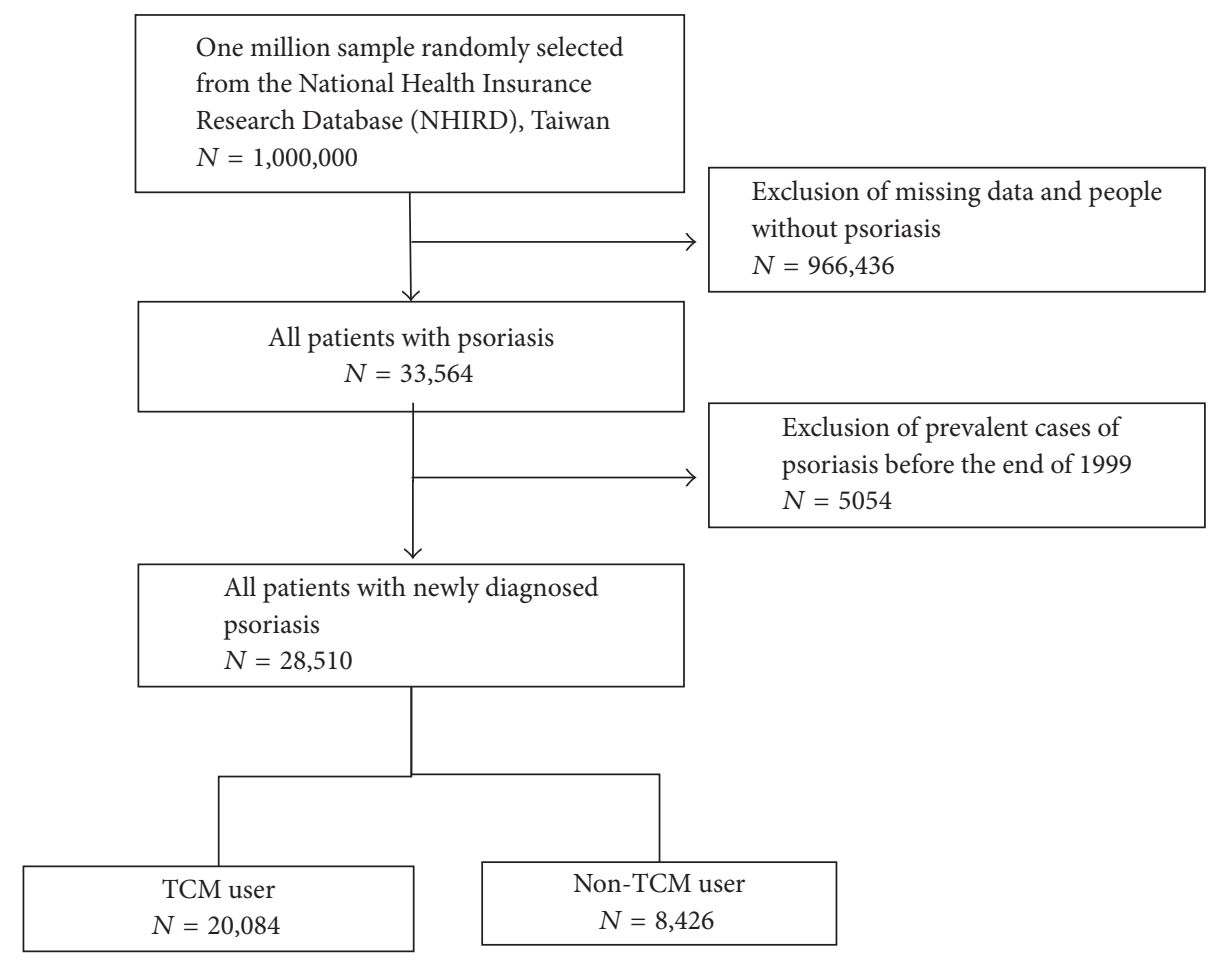

FIGURE 1: Flow recruitment chart of subjects from the one million random samples obtained from the National Health Insurance Research Database (NHIRD) from 2000 to 2010 in Taiwan.

showed the mean and standard deviation (SD) for age and number and percentage for sex and comorbidity. To assess the distribution difference between TCM and non-TCM users, the t-test was used for continuous variable (age) and chisquare test was used for category variables (sex, occupation, urbanization, and major disease categories/diagnosis). The crude and adjusted prevalence rate ratio (PRR) and 95\% confidence intervals (CIs) of those particular diseases for the TCM users compared with non-TCM users were estimated using Poisson regression models. A $p$ value of $<0.05$ was considered as statistically significant.

\section{Results}

There were 28,510 patients who were newly diagnosed with psoriasis (Table 1). Among them, 20,084 (70.4\%) patients ever used TCM outpatient services. The majority (54.9\%) of the TCM users were female. The mean age of TCM users was younger than that of non-TCM users ( 30.0 versus 34.5 years old). The majority (59.2\%) of the TCM users were whitecollar workers. Most of the TCM users resided in urbanized areas. The average time between onset of psoriasis and the first visit to a TCM clinic was 12.0 months.

Regarding the treatment modalities given to patients with psoriasis, 11,609 (57.8\%) patients received only CHM, while 214 (1.1\%) patients were treated by acupuncture or Chinese traumatology only and 8,261 (41.1\%) patients received the combination of both treatments. More than half of the patients $(n=11,037 ; 55.0 \%)$ visited TCM clinics for more than 6 times per year (Table 2).

To investigate the prescription patterns of the Chinese herbal remedies, we conducted a comprehensive analysis and identified ten most commonly prescribed Chinese herbal formulas (Table 3) and single herbs (Table 4), respectively. The most frequently prescribed Chinese herbal formula was Win-qing-yin (Warm Clearing Beverage). Regarding the single herbs for the treatment of psoriasis, Bai-xian-pi (Cortex Dictamni; Dictamnus dasycarpus Turcz.) was the most commonly prescribed single herb.

To further investigate the core prescription patterns, we conducted the network analysis. We found that Mu-danpi (Cortex Moutan; Paeonia suffruticosa Andrews), Winqing-yin (Warm Clearing Beverage), Zi-cao (Radix Lithospermi; Lithospermum erythrorhizon Siebold \& Zucc.; Arnebia euchroma (Royle) I.M.Johnst.; Arnebia guttata Bunge), Baixian-pi (Cortex Dictamni; Dictamnus dasycarpus Turcz.), and Di-fu-zi (Fructus Kochiae; Kochia scoparia (L.) Schrad.) composed the core prescription patterns of $\mathrm{CHM}$ to treat psoriasis patients (Figure 2).

Comparing the disease prevalence rate ratio of TCM and non-TCM group, we found that psoriasis patients with certain disease tended to consult TCM service more than Western medicine consultation (Table 5). Specifically, psoriasis patients preferred to visit TCM doctors when they had metabolic syndrome, hepatitis, rheumatoid arthritis, alopecia areata, Crohn's disease, cancer, depression, fatty liver, chronic airway obstruction, sleep disorder, and allergic rhinitis. 
TABLE 1: Demographic characteristics of TCM and non-TCM users among patients with psoriasis from 2000 to 2010 in Taiwan.

\begin{tabular}{|c|c|c|c|c|c|}
\hline \multirow{2}{*}{ Variable } & \multicolumn{2}{|c|}{ Non-TCM users } & \multicolumn{2}{|c|}{ TCM users } & \multirow{2}{*}{$p$ value } \\
\hline & $N$ & $(\%)$ & $N$ & $(\%)$ & \\
\hline Number of cases & 8426 & 29.6 & 20084 & 70.4 & \\
\hline $\operatorname{Sex}^{\dagger}$ & & & & & $<0.0001$ \\
\hline Female & 3457 & 41.0 & 11035 & 54.9 & \\
\hline Male & 4969 & 59.0 & 9049 & 45.1 & \\
\hline $\operatorname{Age}^{\dagger}, y$ & & & & & $<0.0001$ \\
\hline$<25$ & 3560 & 42.3 & 9601 & 47.8 & \\
\hline $25-35$ & 1275 & 15.1 & 3681 & 18.3 & \\
\hline $35-65$ & 2451 & 29.1 & 5554 & 27.7 & \\
\hline$\geqq 65$ & 1140 & 13.5 & 1248 & 6.2 & \\
\hline Mean $(\mathrm{SD})^{\#}$ & $34.5(22.7)$ & & $30.0(18.7)$ & & $<0.0001$ \\
\hline Occupation $^{\dagger}$ & & & & & $<0.0001$ \\
\hline White collar $\$$ & 4716 & 56.0 & 11892 & 59.2 & \\
\hline Blue collar ${ }^{*}$ & 2475 & 29.4 & 5805 & 28.9 & \\
\hline Others $^{\ddagger}$ & 1235 & 14.7 & 2387 & 11.9 & \\
\hline Urbanization $^{\dagger}$ & & & & & 0.0090 \\
\hline 1 (highest) & 2487 & 29.5 & 5992 & 29.8 & \\
\hline 2 & 2467 & 29.3 & 6007 & 29.9 & \\
\hline 3 & 1514 & 18.0 & 3773 & 18.8 & \\
\hline $4+$ (lowest) & 1958 & 23.2 & 4312 & 21.5 & \\
\hline $\begin{array}{l}\text { Interval between the diagnosis of psoriasis } \\
\text { and the first visit to a TCM clinic, months, } \\
\text { median (IQR) }\end{array}$ & & & $12.0(28.4)$ & & \\
\hline
\end{tabular}

TABLE 2: Frequency distribution of TCM clinic visits and treatment modalities among TCM users from 2000 to 2010 in Taiwan.

\begin{tabular}{|c|c|c|c|c|}
\hline $\begin{array}{l}\text { Number of } \\
\text { TCM visits }\end{array}$ & $\begin{array}{l}\text { Only Chinese herbal } \\
\text { medicine } \\
N=11609(57.8 \%)\end{array}$ & $\begin{array}{c}\text { Only Acupuncture or } \\
\text { traumatology } \\
N=214(1.1 \%)\end{array}$ & $\begin{array}{c}\text { Combination of both } \\
\text { treatments } \\
N=8261(41.1 \%)\end{array}$ & $\begin{array}{c}\text { Total } \\
N=20084(100 \%)\end{array}$ \\
\hline $1-3$ & 4753 (40.9) & 201 (93.9) & $924(11.2)$ & $5878(29.3)$ \\
\hline $4-6$ & 1903 (16.4) & $10(4.7)$ & $1256(15.2)$ & 3169 (15.7) \\
\hline$>6$ & $4953(42.7)$ & $3(1.4)$ & $6081(73.6)$ & $11037(55.0)$ \\
\hline
\end{tabular}

\section{Discussion}

This nationwide population-based study analyzed a cohort of one million beneficiaries from the NHIRD to investigate the TCM usage among patients with psoriasis. We found that approximately $70.4 \%$ of the patients with psoriasis visited TCM clinics. Psoriasis patients with female gender, a younger age, residency in urbanized area, and white collar had a tendency to consult TCM service. The most commonly adopted TCM treatment is CHM. We also identified the ten most common prescribed Chinese herbal formulas and single herbs for the treatment of psoriasis. Wen-qing-yin and Baixian-pi were the most commonly used TCM herbal formula and single herb, respectively. Overall, our study provided valuable information and added value to the existing knowledge regarding TCM treatment for patients with psoriasis.

In our study, women, compared with men, were more likely to use TCM. We found that the highest TCM utilization rate was young people. Several surveys also showed similar findings [14, 32]; women and younger people had a higher usage of TCM. There was also a tendency for TCM users to reside in higher urbanized area. This may be because highly urbanized areas have a high density of TCM doctors in Taiwan [32].

Patients with psoriasis utilized healthcare services significantly more often than those without psoriasis [33], and we found that half of patients with psoriasis used TCM. In this study, patients visited TCM services on an average of 


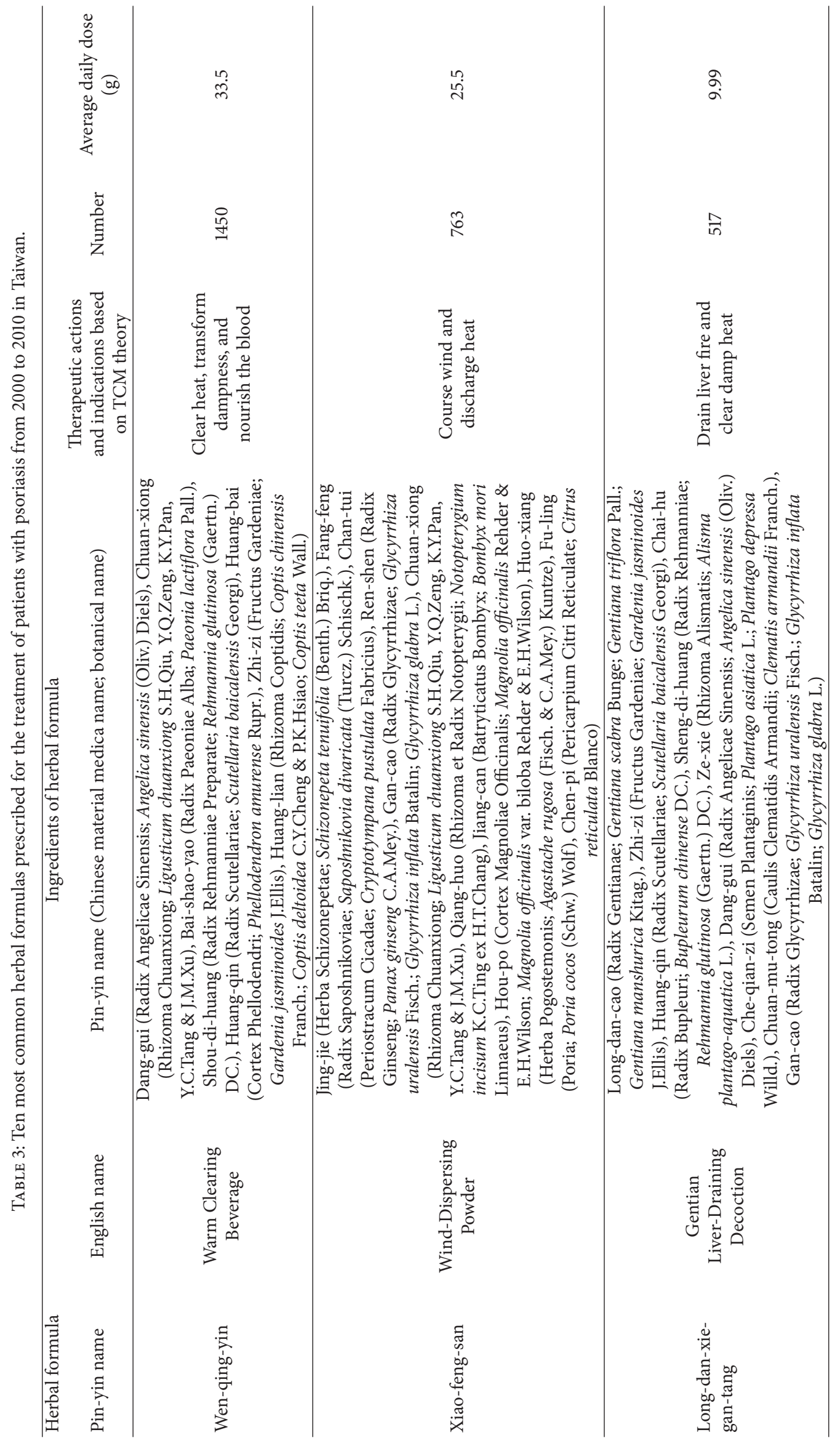




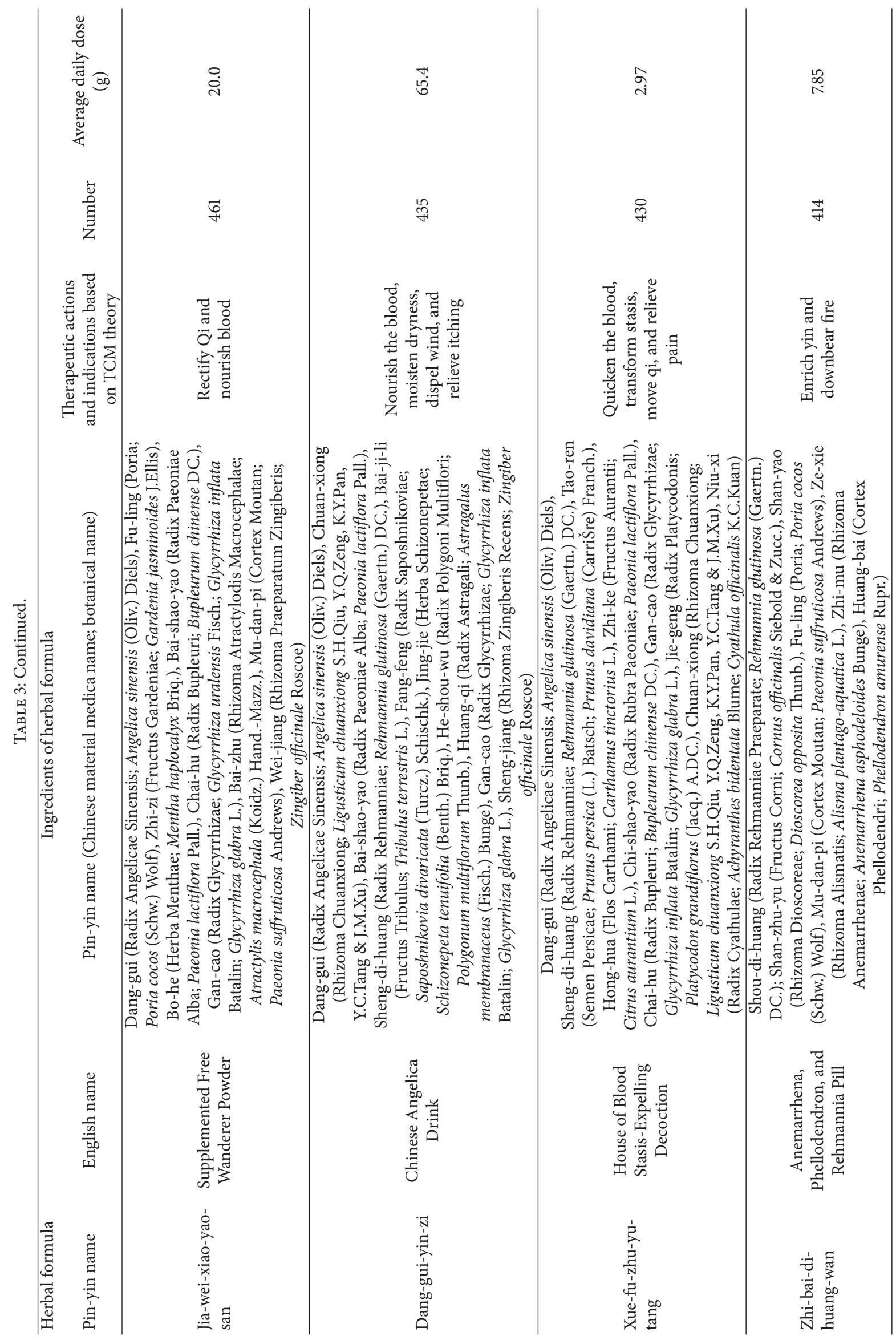







TABLE 4: Top ten most common single herbs prescribed for the treatment of patients with psoriasis from 2000 to 2010 in Taiwan.

\begin{tabular}{|c|c|c|c|c|c|}
\hline \multirow[b]{2}{*}{ Pin-yin name } & \multicolumn{3}{|c|}{ Single herb } & \multirow[b]{2}{*}{ Number } & \multirow[b]{2}{*}{$\begin{array}{l}\text { Average daily } \\
\text { dose (g) }\end{array}$} \\
\hline & $\begin{array}{l}\text { Chinese materia } \\
\text { medica name }\end{array}$ & Botanical name & $\begin{array}{l}\text { Therapeutic actions and } \\
\text { indications based on TCM } \\
\text { theory }\end{array}$ & & \\
\hline Bai-xian-pi & $\begin{array}{l}\text { Cortex } \\
\text { Dictamni }\end{array}$ & $\begin{array}{l}\text { Dictamnus dasycarpus } \\
\text { Turcz. }\end{array}$ & $\begin{array}{l}\text { Clearing heat, drying dampness, } \\
\text { dispelling wind, and resolving } \\
\text { toxin }\end{array}$ & 783 & 1.52 \\
\hline Mu-dan-pi & Cortex Moutan & $\begin{array}{l}\text { Paeonia suffruticosa } \\
\text { Andrews }\end{array}$ & $\begin{array}{l}\text { Clearing heat, cooling the blood, } \\
\text { quickening the blood, and } \\
\text { dispersing stasis }\end{array}$ & 721 & 3.88 \\
\hline Sheng-di-huang & $\begin{array}{c}\text { Radix } \\
\text { Rehmanniae }\end{array}$ & $\begin{array}{l}\text { Rehmannia glutinosa } \\
\quad \text { (Gaertn.) DC. }\end{array}$ & $\begin{array}{l}\text { Clearing heat, cooling the blood, } \\
\text { nourishing yin, and engendering } \\
\text { liquid }\end{array}$ & 602 & 5.98 \\
\hline Tu-fu-ling & $\begin{array}{c}\text { Rhizoma } \\
\text { Smilacis Glabrae }\end{array}$ & Smilax glabra Roxb. & $\begin{array}{l}\text { Resolving toxin and drying } \\
\text { dampness }\end{array}$ & 502 & 7.28 \\
\hline Dan-shen & $\begin{array}{l}\text { Radix Salviae } \\
\text { Miltiorrhizae }\end{array}$ & $\begin{array}{l}\text { Salvia miltiorrhiza } \\
\text { Bunge }\end{array}$ & $\begin{array}{l}\text { Clearing and quickening the } \\
\text { blood and regulating } \\
\text { menstruation }\end{array}$ & 479 & 1.30 \\
\hline Zi-cao & $\begin{array}{l}\text { Radix } \\
\text { Lithospermi }\end{array}$ & $\begin{array}{c}\text { Lithospermum } \\
\text { erythrorhizon Siebold } \\
\text { \& Zucc.; Arnebia } \\
\text { euchroma (Royle) } \\
\text { I.M.Johnst.; Arnebia } \\
\text { guttata Bunge }\end{array}$ & $\begin{array}{l}\text { Clearing and quickening the } \\
\text { blood, resolving toxin, and } \\
\text { outthrusting papules }\end{array}$ & 469 & 1.38 \\
\hline Di-fu-zi & Fructus Kochiae & $\begin{array}{l}\text { Kochia scoparia (L.) } \\
\text { Schrad. }\end{array}$ & $\begin{array}{l}\text { Clearing heat, drying dampness, } \\
\text { and relieving itching }\end{array}$ & 440 & 8.44 \\
\hline Lian-qiao & $\begin{array}{l}\text { Fructus } \\
\text { Forsythiae }\end{array}$ & $\begin{array}{l}\text { Forsythia suspensa } \\
\text { (Thunb.) Vahl }\end{array}$ & $\begin{array}{c}\text { Clearing heat, resolving toxin, } \\
\text { coursing wind, and dispersing } \\
\text { heat }\end{array}$ & 440 & 23.5 \\
\hline Chi-shao-yao & $\begin{array}{l}\text { Radix Rubra } \\
\text { Paeoniae }\end{array}$ & Paeonia lactiflora Pall. & $\begin{array}{c}\text { Clearing heat, cooling the blood, } \\
\text { dispersing stasis, and relieving } \\
\text { pain }\end{array}$ & 402 & 1.31 \\
\hline Jin-yin-hua & Flos Lonicerae & $\begin{array}{l}\text { Lonicera japonica } \\
\text { Thunb. }\end{array}$ & $\begin{array}{c}\text { Clearing heat, resolving toxin, } \\
\text { coursing wind, and dispersing } \\
\text { heat }\end{array}$ & 392 & 16.8 \\
\hline
\end{tabular}

12.0 months after initial diagnosis of psoriasis. Probably most patients usually used Western medicine as the first choice initially. If patients were unsatisfied with conventional therapy, recurrent symptoms, or high costs of biological agents, they might further seek adjunctive TCM consultation $[34,35]$.

In accordance with other studies in atopic dermatitis or urticaria $[13,36], \mathrm{CHM}$ is the most common treatment approaches. We found that only very few patients received acupuncture therapy. Regarding the visiting frequency, a majority of the patients used TCM service for more than 6 times, which might be due to the confidence in symptoms relief or effectiveness perceived by users. However, for those who received both treatment modalities, they tended to use TCM services more frequently. It is possible that those patients who chose a combination of CHM and acupuncture or traumatology therapy had complicated situations that required more frequent treatment.

Psoriasis is not a disease merely affecting skin. We found that patients with psoriasis were associated with a high degree of incidence rate ratio for visiting TCM clinics when they suffered from various medical comorbidities. Previous investigations were consistent with our findings that psoriasis patients had a broad spectrum of disease category in their clinical visits $[2,37]$.

According to the theory of traditional medicine, psoriasis is further identified by different patterns, such as blood heat, heat toxin, damp heat, blood stasis, and blood dryness. Our network analysis in Figure 2 included the top 50 combinations of herbal formulas and herbs for psoriasis patients. According to the theory of TCM theory, this core pattern can clear heat, cool the blood, nourish the blood, dispel wind, and dry dampness.

Wen-qing-yin was the most frequently prescribed formula for psoriasis. It was originally documented in an ancient literature as "Wan Bing Hui Chun." This formula is composed of Si-wu-tang (Four Agents Decoction) and Huang-lian-jiedu-tang (Coptis Toxin-Resolving Decoction). Together in this formula, they can clear heat, transform dampness, and nourish the blood based on the TCM theory. In a previous report, Wen-qing-yin could inhibit the induction phase of various kinds of delayed type hypersensitivity and local graftversus-host reactions [38]. The second commonly prescribed 


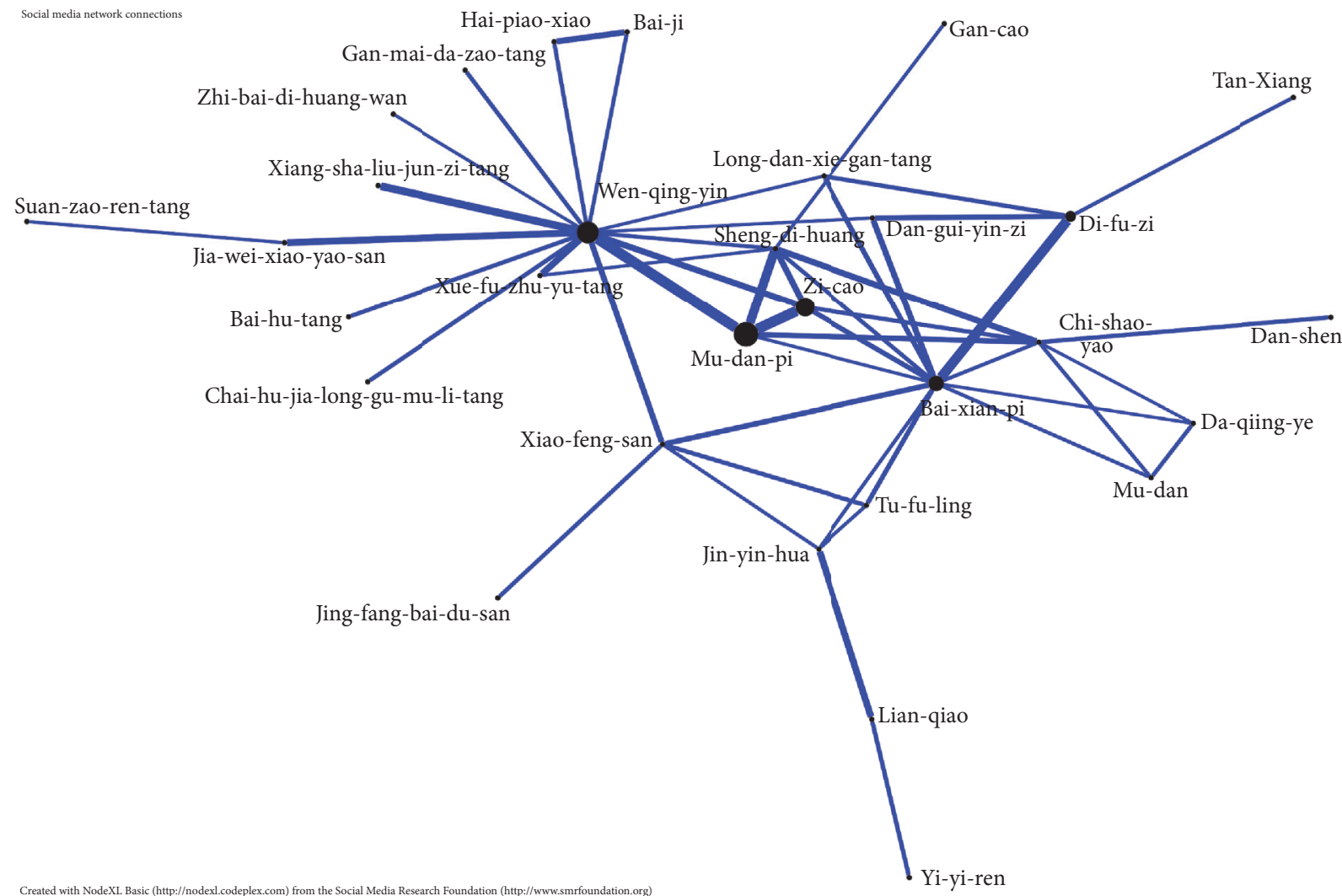

Figure 2: The top 50 combinations of herbal formulas and single herbs for psoriasis patients were analyzed through open-sourced freeware NodeXL. The core prescription pattern was Mu-dan-pi, Wen-qing-yin, Zi-cao, Bai-xian-pi, and Di-fu-zi.

herbal formula is Xiao-feng-san (Wind-Dispersing Powder). It has long been used to treat skin problems in clinical practice such as atopic dermatitis [13] or urticaria [36]. In a clinical trial conducted in our institution, Xiao-feng-san was found to improve refractory atopic dermatitis symptom and with no side effects [39]. In basic studies, Xiao-fengsan as an antiallergic drug was reported to inhibit IgE dependent histamine release from the cultured mast cells [40]. Xiao-feng-san might also correct the Th1/Th2 balance by preventing the increase in interleukin- $4 \mathrm{mRNA}$ expression and the decrease in interferon-gamma mRNA expression to inhibit dermatitis [41]. Long-dan-xie-gen-tang (Gentian Liver-Draining Decoction) is the third most common formula. In clinical practice, Long-dan-xie-gen-tang is commonly prescribed to subjects with chronic hepatitis [42], atopic dermatitis [13], and insomnia [43] in Taiwan. In TCM viewpoint, it can drain fire and clear damp heat. Besides the symptoms of skin, patients with psoriasis often suffered from sleep problems $[2,44]$. Whether Long-dan-xie-gen-tang has direct effect on skin or indirect efficacy on comorbidity, such as insomnia, deserves further investigation.

Bai-xian-pi (Cortex Dictamni; Dictamnus dasycarpus Turcz.) was the most commonly prescribed single herb. It can clear heat and dry dampness, dispel wind, and resolve toxin. Among the prescribed single herbs, seven of top ten single herbs belonged to the category of "clearing heat." Tropical treatment of methanol extract of Dictamnus dasycarpus Turcz. root bark on dermatitis mice could effectively inhibit skin thickness, hyperplasia, and edema [45]. The anti-inflammatory effects of Dictamnus dasycarpus Turcz. may reduce the level of $\beta$-hexosaminidase and histamine release [46]. Mu-dan-pi (Cortex Moutan; Paeonia suffruticosa Andrews) was used to clear heat and cool the blood in TCM. Previous study found that the water extract of Moutan Cortex not only inhibited $\beta$-hexosaminidase and tumor necrosis factor- $\alpha$ release in IgE-mediated DNP-BSAstimulated RBL-2H3 cells but also improved the compound 48/80-induced allergic reactions in a mouse model [47]. $\mathrm{Zi}$ cao (Radix Lithospermi; Lithospermum erythrorhizon Siebold \& Zucc.; Arnebia euchroma (Royle) I.M.Johnst.; Arnebia guttata Bunge) has been used to clear blood heat and has shown an anti-inflammatory effect in experimental studies [48]. Its ingredient, shikonin, was found to suppress IL-17 signaling in keratinocytes [49]. Di-fu-zi (Fructus Kochiae; Kochia scoparia (L.) Schrad.) has been used to clear heat and dry dampness. It was reported to ameliorate dermatitis via inhibition of the production of proinflammatory cytokines [50]. Sheng-di-huang (Radix Rehmanniae; Rehmannia glutinosa (Gaertn.) DC.) polysaccharides extract was found to increase skin glutathione, superoxide dismutase, catalase, and 
TABLE 5: Prevalence rate ratio of diseases between non-TCM and TCM users.

\begin{tabular}{|c|c|c|c|c|c|c|}
\hline & \multicolumn{2}{|c|}{$\begin{array}{c}\text { Non-TCM } \\
N=8426\end{array}$} & \multicolumn{2}{|c|}{$\begin{array}{c}\text { TCM } \\
N=20084\end{array}$} & \multicolumn{2}{|c|}{ Compared to non-TCM } \\
\hline & $n$ & $\%$ & $n$ & $\%$ & Crude PRR & Adjusted $\mathrm{PRR}^{\dagger}$ \\
\hline \multicolumn{7}{|l|}{ Metabolic syndrome } \\
\hline Hypertension & 1817 & 21.6 & 3173 & 15.8 & $0.73(0.70-0.77)^{* * *}$ & $1.18(1.11-1.26)^{* * *}$ \\
\hline Diabetes & 1000 & 11.9 & 1727 & 8.6 & $0.72(0.69-0.76)^{* * *}$ & $1.14(1.05-1.24)^{* *}$ \\
\hline Hyperlipidemia & 1221 & 14.5 & 2666 & 13.3 & $0.92(0.87-0.96)^{* * *}$ & $1.29(1.20-1.39)^{* * *}$ \\
\hline Heart disease & 1379 & 16.4 & 2707 & 13.5 & $0.82(0.78-0.86)^{* * *}$ & $1.20(1.11-1.28)^{* * *}$ \\
\hline \multicolumn{7}{|l|}{ Infections } \\
\hline Tuberculosis & 153 & 1.82 & 198 & 0.99 & $0.54(0.51-0.58)^{* * *}$ & $0.94(0.74-1.19)$ \\
\hline Hepatitis B & 376 & 4.46 & 1193 & 5.94 & $1.33(1.25-1.42)^{* * *}$ & $1.46(1.29-1.65)^{* * *}$ \\
\hline Hepatitis C & 184 & 2.18 & 435 & 2.17 & $0.99(0.92-1.06)$ & $1.39(1.16-1.68)^{* * *}$ \\
\hline \multicolumn{7}{|l|}{ Auto immune disorder } \\
\hline Rheumatoid arthritis & 15 & 0.18 & 48 & 0.24 & $1.34(1.22-1.48)^{* * *}$ & $1.93(1.03-3.60)^{*}$ \\
\hline Systemic lupus erythematosus & 16 & 0.19 & 25 & 0.12 & $0.66(0.60-0.71)^{* * *}$ & $0.59(0.29-1.17)$ \\
\hline Vitiligo & 40 & 0.47 & 123 & 0.61 & $1.29(1.18-1.41)^{* * *}$ & $1.11(0.77-1.62)$ \\
\hline Pemphigoid & 16 & 0.19 & 16 & 0.08 & $0.42(0.39-0.46)^{* * *}$ & $0.86(0.39-1.89)$ \\
\hline Pemphigus & 15 & 0.18 & 14 & 0.07 & $0.39(0.36-0.43)^{* * *}$ & $0.58(0.26-1.32)$ \\
\hline Alopecia areata & 88 & 1.04 & 297 & 1.48 & $1.42(1.31-1.54)^{* * *}$ & $1.36(1.06-1.75)^{*}$ \\
\hline Crohn's disease & 449 & 5.33 & 1610 & 8.02 & $1.50(1.41-1.60)^{* * *}$ & $1.32(1.18-1.47)^{* * *}$ \\
\hline Cancer & 329 & 3.90 & 597 & 2.97 & $0.76(0.71-0.81)^{* * *}$ & $1.24(1.07-1.44)^{* *}$ \\
\hline \multicolumn{7}{|l|}{ Others } \\
\hline Depression & 379 & 4.50 & 1200 & 5.97 & $1.33(1.24-1.42)^{* * *}$ & $1.41(1.25-1.60)^{* * *}$ \\
\hline Hyperthyroidism & 157 & 1.86 & 400 & 1.99 & $1.07(0.99-1.15)$ & $0.99(0.81-1.21)$ \\
\hline Hypothyroidism & 49 & 0.58 & 153 & 0.76 & $1.31(1.20-1.43)^{* * *}$ & $1.20(0.85-1.70)$ \\
\hline Multiple sclerosis & 5 & 0.06 & 15 & 0.07 & $1.26(1.14-1.39)^{* * *}$ & $1.02(0.35-2.99)$ \\
\hline Fatty liver & 144 & 1.71 & 331 & 1.65 & $0.96(0.90-1.04)$ & $1.30(1.05-1.61)^{* *}$ \\
\hline Chronic airways obstruction & 348 & 4.13 & 452 & 2.25 & $0.54(0.51-0.58)^{* * *}$ & $1.19(1.02-1.39)^{*}$ \\
\hline Sleep disorder & 1046 & 12.4 & 4407 & 21.9 & $1.77(1.68-1.86)^{* * *}$ & $1.77(1.65-1.90)^{* * *}$ \\
\hline Asthma & 1008 & 12.0 & 2303 & 11.5 & $0.96(0.91-1.01)$ & $0.98(0.91-1.06)$ \\
\hline Allergic rhinitis & 1956 & 23.2 & 6687 & 33.3 & $1.43(1.37-1.50)^{* * *}$ & $1.26(1.20-1.33)^{* * *}$ \\
\hline
\end{tabular}

PRR: prevalence rate ratio. ${ }^{\dagger}$ Model adjusted for age, sex, occupation, urbanization, and number of outpatient visits for traditional Chinese medicine. ${ }^{*} p \leq$ $0.05 ;{ }^{* *} p \leq 0.01 ;{ }^{* * *} p \leq 0.001$.

glutathione peroxidase activities and decrease skin malondialdehyde level in ultraviolet $B$ ray treated mice, suggesting that it may be useful for skin diseases [51].

Taken together with the classification and principles of TCM formulas and single herbs, our findings corresponded with the viewpoint of TCM, which believes that the occurrence of psoriasis symptoms is related to the blood heat, blood stasis, and blood dryness. However, it has to be pointed out that future validation on their efficacy and safety is necessary.

The strength of this study at least included the following aspects: First, all residents of Taiwan can access the NHI system with low cost and convenience, and thus the accessibility of healthcare, either Western or Chinese medicine, is high. Second, all patients with psoriasis were included in this study and this nationwide population-based study comprehensively included all the prescriptions for psoriasis patients. These TCM formulas and single herbs may provide some thought in the exploration of better treatment options.
Some caveats in this study merit comments. First, this study did not include topical Chinese herbal ointments or herbal bath, which were not the forms of TCM reimbursed by the NHI program. The NHI program only covers TCM prescriptions manufactured by GMP-certified pharmaceutical companies in Taiwan. Future study to explore the topical herbal products is necessary. Second, the severity of psoriasis and efficacy of CHM were not available in this database. Judging from the fact that $70.4 \%$ of the patients with psoriasis used TCM service, it is necessary to conduct clinical trials to evaluate the efficacy and safety of these prescriptions.

\section{Conclusion}

This study is the first large-scale survey to analyze TCM utilization patterns among patients with psoriasis in Taiwan. Patients with psoriasis often sought help from TCM 
treatment. Wen-qing-yin was the most frequently prescribed Chinese herbal formula, while Bai-xian-pi was the most common single herb. Future clinical trials and pharmacological investigations could be developed based on the findings of this study.

\section{Disclosure}

The interpretation and conclusions contained herein do not represent those of National Health Insurance Administration, Ministry of Health and Welfare, or National Health Research Institutes.

\section{Competing Interests}

The authors declare that they have no conflict of interests.

\section{Authors' Contributions}

Shu-Wen Weng, Jaung-Geng Lin, and Hung-Rong Yen conceptualized the study. Yu-Chiao Wang performed the statistical analysis. Bor-Chyuan Chen, Chun-Kai Liu, Mao-Feng Sun, Jaung-Geng Lin, and Hung-Rong Yen contributed to the interpretation of TCM data. Bor-Chyuan Chen interpreted the pharmacological mechanisms. Ching-Mao Chang conducted the network analysis of the core prescription pattern. Shu-Wen Weng, Bor-Chyuan Chen, and Hung-Rong Yen drafted the manuscript. Jaung-Geng Lin and Hung-Rong Yen finalized the manuscript. Shu-Wen Weng and Bor-Chyuan Chen have equal contribution.

\section{Acknowledgments}

This study was supported by China Medical University under the Aim for Top University Plan of the Ministry of Education, Taiwan. This study was also supported in part by the Taiwan Ministry of Health and Welfare Clinical Trial and Research Center of Excellence (MOHW105-TDUB-212-133019). This study was based in part on data from the National Health Insurance Research Database, provided by the National Health Insurance Administration, Ministry of Health and Welfare, and managed by National Health Research Institutes.

\section{References}

[1] R. Parisi, D. P. Symmons, C. E. Griffiths, D. M. Ashcroft, and Identification and Management of Psoriasis and Associated ComorbidiTy (IMPACT) Project Team, "Global epidemiology of psoriasis: a systematic review of incidence and prevalence," The Journal of Investigative Dermatology, vol. 133, pp. 377-385, 2013.

[2] T.-F. Tsai, T.-S. Wang, S.-T. Hung et al., "Epidemiology and comorbidities of psoriasis patients in a national database in Taiwan," Journal of Dermatological Science, vol. 63, no. 1, pp. 4046, 2011.

[3] J. Vanderpuye-Orgle, Y. Zhao, J. Lu et al., "Evaluating the economic burden of psoriasis in the United States," Journal of the American Academy of Dermatology, vol. 72, no. 6, pp. 961.e5967.e5, 2015.

[4] K.-C. Chen, S.-T. Hung, C.-W. W. Yang, T.-F. Tsai, and C.-H. Tang, "The economic burden of psoriatic diseases in Taiwan," Journal of Dermatological Science, vol. 75, no. 3, pp. 183-189, 2014.

[5] W.-H. Boehncke and M. P. Schön, "Psoriasis," The Lancet, vol. 386, no. 9997, pp. 983-994, 2015.

[6] L. Barnes, G. Kaya, and V. Rollason, “Topical corticosteroidinduced skin atrophy: a comprehensive review," Drug Safety, vol. 38, no. 5, pp. 493-509, 2015.

[7] J. G. Schlager, S. Rosumeck, R. N. Werner et al., "Topical treatments for scalp psoriasis," The Cochrane Database of Systematic Reviews, no. 2, Article ID CD009687, 2016.

[8] K. Papp, J. C. Cather, L. Rosoph et al., "Efficacy of apremilast in the treatment of moderate to severe psoriasis: a randomised controlled trial," The Lancet, vol. 380, no. 9843, pp. 738-746, 2012.

[9] E. Archier, S. Devaux, E. Castela et al., "Carcinogenic risks of Psoralen UV-A therapy and Narrowband UV-B therapy in chronic plaque psoriasis: a systematic literature review," Journal of the European Academy of Dermatology and Venereology, vol. 26, no. 3, pp. 22-31, 2012.

[10] R. Conway, C. Low, R. J. Coughlan, M. J. O’Donnell, and J. J. Carey, "Risk of liver injury among methotrexate users: a metaanalysis of randomised controlled trials," Seminars in Arthritis and Rheumatism, vol. 45, no. 2, pp. 156-162, 2015.

[11] A. Maza, H. Montaudié, E. Sbidian et al., "Oral cyclosporin in psoriasis: a systematic review on treatment modalities, risk of kidney toxicity and evidence for use in non-plaque psoriasis," Journal of the European Academy of Dermatology and Venereology, vol. 25, supplement 2, pp. 19-27, 2011.

[12] O. D. van Cranenburgh, J. de Korte, M. A. G. Sprangers, M. A. de Rie, and E. M. A. Smets, "Satisfaction with treatment among patients with psoriasis: a web-based survey study," The British Journal of Dermatology, vol. 169, no. 2, pp. 398-405, 2013.

[13] J.-F. Lin, P.-H. Liu, T.-P. Huang et al., "Characteristics and prescription patterns of traditional chinese medicine in atopic dermatitis patients: ten-year experiences at a medical center in taiwan," Complementary Therapies in Medicine, vol. 22, no. 1, pp. 141-147, 2014.

[14] C.-Y. Huang, W.-Y. Lai, M.-F. Sun et al., "Prescription patterns of traditional Chinese medicine for peptic ulcer disease in Taiwan: a nationwide population-based study," Journal of Ethnopharmacology, vol. 176, pp. 311-320, 2015.

[15] M.-C. Huang, F.-T. Pai, C.-C. Lin et al., "Characteristics of traditional Chinese medicine use in patients with rheumatoid arthritis in Taiwan: a nationwide population-based study," Journal of Ethnopharmacology, vol. 176, pp. 9-16, 2015.

[16] A. L. Lee, B. C. Chen, C. H. Mou, M. F. Sun, and H. R. Yen, "Association of traditional Chinese medicine therapy and the risk of vascular complications in patients with type II diabetes mellitus: a nationwide, retrospective, Taiwaneseregistry, cohort study," Medicine, vol. 95, no. 3, Article ID e2536, 2016.

[17] A. S.-Y. Lien, Y.-D. Jiang, C.-H. Mou, M.-F. Sun, B.-S. Gau, and H.-R. Yen, "Integrative traditional Chinese medicine therapy reduces the risk of diabetic ketoacidosis in patients with type 1 diabetes mellitus," Journal of Ethnopharmacology, vol. 191, pp. 324-330, 2016.

[18] H.-R. Yen, Y.-Y. Chen, T.-P. Huang et al., "Prescription patterns of Chinese herbal products for patients with uterine fibroid 
in Taiwan: a nationwide population-based study," Journal of Ethnopharmacology, vol. 171, pp. 223-230, 2015.

[19] T. Fleischer, T.-T. Chang, J.-H. Chiang, C.-M. Chang, C.-Y. Hsieh, and H.-R. Yen, "Adjunctive Chinese Herbal Medicine therapy improves survival of patients with chronic myeloid leukemia: a nationwide population-based cohort study," Cancer Medicine, vol. 5, no. 4, pp. 640-648, 2016.

[20] H. R. Yen, W. Y. Lai, C. H. Muo, and M. F. Sun, "Characteristics of traditional Chinese medicine use in pediatric cancer patients: a nationwide, retrospective, Taiwanese-registry, populationbased study," Integrative Cancer Therapies, 2016.

[21] C. S. Zhang, L. Yang, A. L. Zhang et al., "Is oral Chinese herbal medicine beneficial for psoriasis vulgaris? A meta-analysis of comparisons with acitretin," The Journal of Alternative and Complementary Medicine, vol. 22, no. 3, pp. 174-188, 2016.

[22] J. J. Yu, C. S. Zhang, A. L. Zhang, B. May, C. C. Xue, and C. Lu, "Add-on effect of Chinese herbal medicine bath to phototherapy for psoriasis vulgaris: a systematic review," Evidence-Based Complementary and Alternative Medicine, vol. 2013, Article ID 673078, 14 pages, 2013.

[23] Y.-K. Lin, L.-C. See, Y.-H. Huang et al., "Efficacy and safety of Indigo naturalis extract in oil (Lindioil) in treating nail psoriasis: a randomized, observer-blind, vehicle-controlled trial," Phytomedicine, vol. 21, no. 7, pp. 1015-1020, 2014.

[24] Y. Yan, W. Liu, P. Andres et al., "Exploratory clinical trial to evaluate the efficacy of a topical traditional Chinese herbal medicine in psoriasis vulgaris," Evidence-Based Complementary and Alternative Medicine, vol. 2015, Article ID 719641, 6 pages, 2015.

[25] E. T. Landis, S. A. Davis, S. R. Feldman, and S. Taylor, "Complementary and alternative medicine use in dermatology in the United States," Journal of Alternative and Complementary Medicine, vol. 20, no. 5, pp. 392-398, 2014.

[26] NHIA, National Health Insurance Annual Report 2015-2016, National Health Insurance Administration, Ministry of Health and Welfare, Taipei, Taiwan, 2015.

[27] C. C. Chang, Y. C. Lee, C. C. Lin et al., "Characteristics of traditional chinese medicine usage in patients with stroke in taiwan: a nationwide population-based study," Journal of Ethnopharmacology, vol. 186, pp. 311-321, 2016.

[28] D. Bensky, S. Clavey, and E. Stoger, Chinese Herbal Medicine: Materia Medica, Eastland Press, Seattle, Wash, USA, 3rd edition, 2004.

[29] V. Scheid, D. Bensky, A. Ellis, and R. Barolet, Chinese Herbal Medicine: Formulas \& Strategies, Eastland Press, 2009.

[30] K. Chan, D. Shaw, M. S. J. Simmonds et al., "Good practice in reviewing and publishing studies on herbal medicine, with special emphasis on traditional Chinese medicine and Chinese materia medica," Journal of Ethnopharmacology, vol. 140, no. 3, pp. 469-475, 2012.

[31] C. M. Chang, H. T. Chu, Y. H. Wei et al., "The core pattern analysis on Chinese herbal medicine for Sjogren's syndrome: a nationwide population-based study," Scientific Reports, vol. 5, article 9541, 2015.

[32] C.-C. Shih, C.-C. Liao, Y.-C. Su, C.-C. Tsai, and J.-G. Lin, "Gender differences in traditional chinese medicine use among adults in Taiwan," PLoS ONE, vol. 7, no. 4, Article ID e32540, 2012.

[33] L.-T. Kao, K.-H. Wang, H.-C. Lin, H.-C. Li, S. Yang, and S.-D. Chung, "Use of health care services by patients with psoriasis: a population-based study," The British Journal of Dermatology, vol. 172, no. 5, pp. 1346-1352, 2015.
[34] E. Ben-Arye, M. Ziv, M. Frenkel, I. Lavi, and D. Rosenman, "Complementary medicine and psoriasis: linking the patient's outlook with evidence-based medicine," Dermatology, vol. 207, no. 3, pp. 302-307, 2003.

[35] G.-W. Kim, J.-M. Park, H.-W. Chin et al., "Comparative analysis of the use of complementary and alternative medicine by Korean patients with androgenetic alopecia, atopic dermatitis and psoriasis," Journal of the European Academy of Dermatology and Venereology, vol. 27, no. 7, pp. 827-835, 2013.

[36] P.-S. Chien, Y.-F. Tseng, Y.-C. Hsu, Y.-K. Lai, and S.-F. Weng, "Frequency and pattern of Chinese herbal medicine prescriptions for urticaria in Taiwan during 2009: analysis of the national health insurance database," BMC Complementary and Alternative Medicine, vol. 13, article 209, 2013.

[37] H. Yeung, J. Takeshita, N. N. Mehta et al., "Psoriasis severity and the prevalence of major medical comorbidity: a populationbased study," JAMA Dermatology, vol. 149, no. 10, pp. 1173-1179, 2013.

[38] H. Mori, M. Fuchigami, N. Inoue, H. Nagai, A. Koda, and I. Nishioka, "Principle of the bark of Phellodendron amurense to suppress the cellular immune response," Planta Medica, vol. 60, no. 5, pp. 445-449, 1994.

[39] H.-M. Cheng, L.-C. Chiang, Y.-M. Jan, G.-W. Chen, and T.-C. $\mathrm{Li}$, "The efficacy and safety of a Chinese herbal product (XiaoFeng-San) for the treatment of refractory atopic dermatitis: a randomized, double-blind, placebo-controlled trial," International Archives of Allergy and Immunology, vol. 155, no. 2, pp. 141-148, 2011.

[40] K. Shichijo and H. Saito, "Effect of Chinese herbal medicines and disodium cromoglycate on IgE-dependent histamine release from mouse cultured mast cells," International Journal of Immunopharmacology, vol. 19, no. 11-12, pp. 677-682, 1998.

[41] X. K. Gao, K. Fuseda, T. Shibata, H. Tanaka, N. Inagaki, and H. Nagai, "Kampo medicines for mite antigen-induced allergic dermatitis in NC/Nga mice," Evidence-Based Complementary and Alternative Medicine, vol. 2, no. 2, pp. 191-199, 2005.

[42] F.-P. Chen, Y.-Y. Kung, Y.-C. Chen et al., "Frequency and pattern of Chinese herbal medicine prescriptions for chronic hepatitis in Taiwan," Journal of Ethnopharmacology, vol. 117, no. 1, pp. 8491, 2008.

[43] F.-P. Chen, M.-S. Jong, Y.-C. Chen et al., "Prescriptions of Chinese herbal medicines for insomnia in Taiwan during 2002," Evidence-Based Complementary and Alternative Medicine, vol. 2011, Article ID 236341, 9 pages, 2011.

[44] M. A. Gupta, F. C. Simpson, and A. K. Gupta, "Psoriasis and sleep disorders: a systematic review," Sleep Medicine Reviews, vol. 29, pp. 63-75, 2016.

[45] H. Kim, M. Kim, H. Kim, G. San Lee, W. G. An, and S. I. Cho, "Anti-inflammatory activities of Dictamnus dasycarpus Turcz., root bark on allergic contact dermatitis induced by dinitrofluorobenzene in mice," Journal of Ethnopharmacology, vol. 149, no. 2, pp. 471-477, 2013.

[46] H.-Y. Han, M. H. Ryu, G. Lee et al., "Effects of Dictamnus dasycarpus Turcz., root bark on ICAM-1 expression and chemokine productions in vivo and vitro study," Journal of Ethnopharmacology, vol. 159, pp. 245-252, 2015.

[47] J.-Y. Kee, A. Inujima, T. Andoh et al., "Inhibitory effect of Moutan Cortex aqueous fraction on mast cell-mediated allergic inflammation," Journal of Natural Medicines, vol. 69, no. 2, pp. 209-217, 2015. 
[48] C. S. Zhang, J. J. Yu, S. Parker et al., "Oral Chinese herbal medicine combined with pharmacotherapy for psoriasis vulgaris: a systematic review," International Journal of Dermatology, vol. 53, no. 11, pp. 1305-1318, 2014.

[49] Y. Xu, X. Xu, X. Gao, H. Chen, and L. Geng, "Shikonin suppresses IL-17-induced VEGF expression via blockage of JAK2/STAT3 pathway," International Immunopharmacology, vol. 19, no. 2, pp. 327-333, 2014.

[50] Y. Y. Choi, M. H. Kim, J. Y. Lee, J. Hong, S.-H. Kim, and W. M. Yang, "Topical application of Kochia scoparia inhibits the development of contact dermatitis in mice," Journal of Ethnopharmacology, vol. 154, no. 2, pp. 380-385, 2014.

[51] Z. Sui, L. Li, B. Liu et al., "Optimum conditions for Radix Rehmanniae polysaccharides by RSM and its antioxidant and immunity activity in UVB mice," Carbohydrate Polymers, vol. 92, no. 1, pp. 283-288, 2013. 


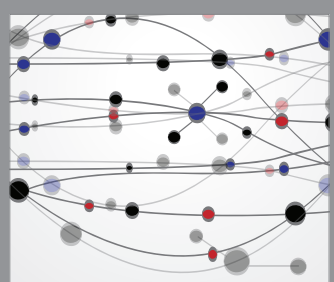

The Scientific World Journal
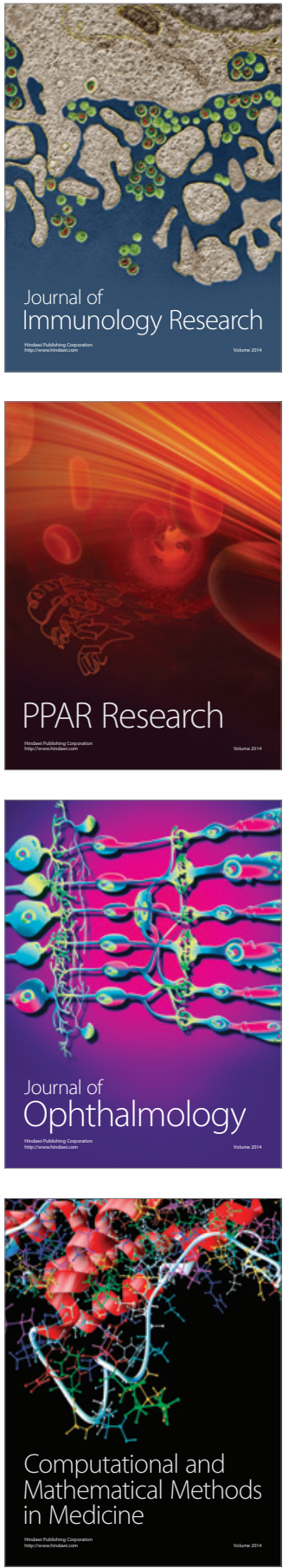

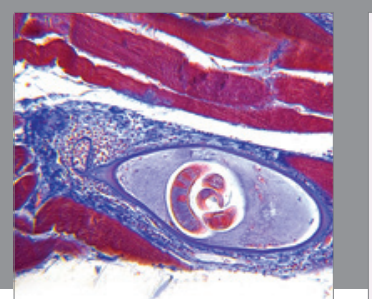

Gastroenterology Research and Practice



\section{Hindawi}

Submit your manuscripts at

http://www.hindawi.com
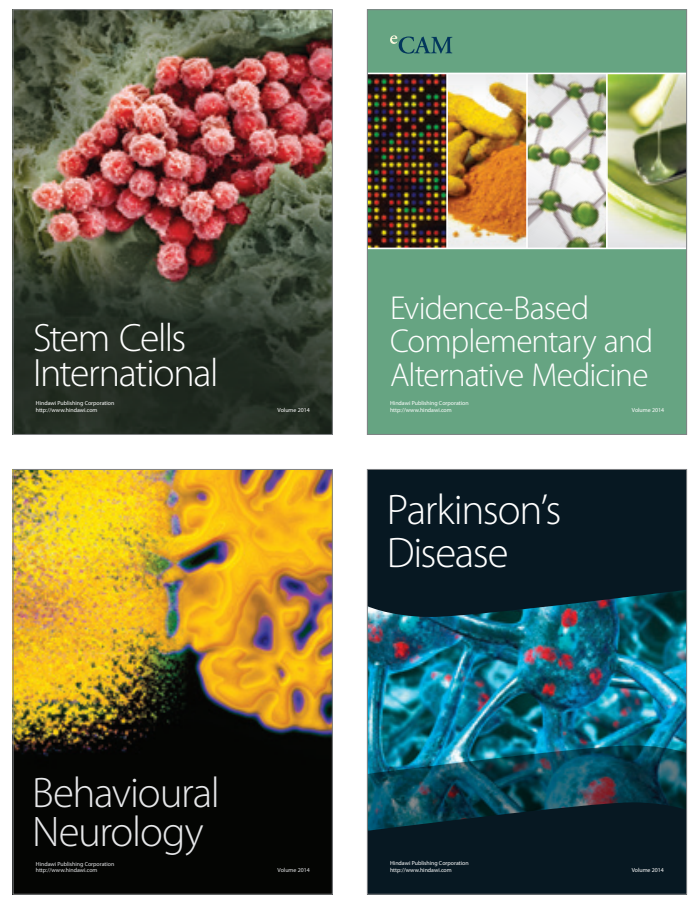


Disease Markers
